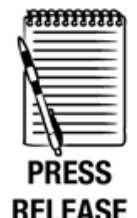

RELEASE

\title{
Urinary trichlorophenol levels and increased risk of attention deficit hyperactivity disorder among US school-aged children
}

\author{
Xiaohui Xu, ${ }^{1}$ Wendy N Nembhard, ${ }^{2}$ Haidong Kan, ${ }^{3}$ Greg Kearney, ${ }^{4}$ Zhi-Jiang Zhang, ${ }^{5}$ \\ Evelyn 0 Talbott $^{6}$
}

${ }^{1}$ Department of Epidemiology and Biostatistics, College of Public Health and Health Professions, University of Florida, Gainesville, Florida, USA ${ }^{2}$ Department of Epidemiology and Biostatistics, College of Public Health, University of South Florida, Tampa, Florida, USA

${ }^{3}$ Department of Environmental Health, School of Public Health, Fudan University, Shanghai,

China

${ }^{4}$ National Center for Environmental Health, Centers for Disease Control and Prevention, Atlanta, Georgia, USA

${ }^{5}$ Department of Epidemiology, School of Public Health, Shanghai Jiao Tong University, Shanghai, China ${ }^{6}$ Department of Epidemiology, Graduate School of Public Health, University of Pittsburgh, Pittsburgh, USA

\section{Correspondence to}

Xiaohui Xu, $101 \mathrm{~S}$ Newell Drive, Room 3119, Gainesville, FL 32610-0182, USA; xhxu@phhp.ufl.edu

Accepted 23 March 2011 Published Online First 3 May 2011

\section{UNLOCKAD}

This paper is freely available online under the BMJ Journals unlocked scheme, see http:// oem.bmj.com/site/about/ unlocked.xhtml

\section{ABSTRACT}

Background Trichlorophenols (TCPs) are organochlorine compounds which are ubiquitous in the environment and well known for their carcinogenic effects. However, little is known about their neurotoxicity in humans.

Objectives Our goal was to examine the association between body burden of TCPs (ie, 2,4,5-TCP and 2,4,6TCP) and attention deficit hyperactivity disorder (ADHD). Methods We calculated ORs and 95\% Cls from logistic regression analyses using data from the 1999-2004 National Health and Nutrition Examination Survey (NHANES) to evaluate the association between urinary TCPs and parent-reported ADHD among 2546 children aged $6-15$ years.

Results Children with low levels $(<3.58 \mu \mathrm{g} / \mathrm{g})$ and high levels $(\geq 3.58 \mu \mathrm{g} / \mathrm{g})$ of urinary 2,4,6-TCP had a higher risk of parent-reported ADHD compared to children with levels below the limit of detection (OR 1.54, 95\% Cl 0.97 to 2.43 and $\mathrm{OR} 1.77,95 \% \mathrm{Cl} 1.18$ to 2.66 , respectively; $\mathrm{p}$ for trend $=0.006$ ) after adjusting for covariates. No association was found between urinary 2,4,5-TCP and parent-reported ADHD.

Conclusion Exposure to TCP may increase the risk of behavioural impairment in children. The potential neurotoxicity of these chemicals should be considered in public health efforts to reduce environmental exposures/ contamination, especially in countries where organochlorine pesticides are still commonly used.

\section{INTRODUCTION}

Trichlorophenols (TCPs) including 2,4,5-trichlorophenol (2,4,5-TCP) and 2,4,6-trichlorophenol (2,4,6TCP) are formed when three chlorine atoms are joined to one phenol molecule. TCPs were historically used as wood preservatives and as intermediates in the chemical synthesis of a variety of pesticides. ${ }^{1}$ Although most uses of TCPs have been prohibited in the USA, they continue to be used in the production of some fungicides. ${ }^{2}$ TCPs are also released into the environment as by-products during the manufacture of other chlorinated aromatic compounds and during the chlorination of drinking water or waste water. TCPs are also the metabolites of several organochlorine chemicals including chlorobenzenes, hexachlorocyclohexanes and chlorophenoxy acids, and are present in the air, surface water, soil and sediments. ${ }^{3}$ Therefore, general population exposure to TCPs can occur through ingestion of food and water or inhalation of air contaminated by TCPs or other organochlorine chemicals.

\section{What this paper adds}

- Trichlorophenols (TCPs) are organochlorine compounds which are ubiquitous in the environment, but there is little epidemiological evidence about their toxic effects on children's neurodevelopment.

- We examined whether background exposure to these chemicals was associated with attention deficit hyperactivity disorder (ADHD) among US children.

- The results revealed that a higher body burden of 2,4,6-TCP was significantly associated with a higher prevalence of ADHD.

Measuring individual exposure to TCPs is challenging due to the many potential exposure pathways. Urinary excretion of TCPs is an indication of exposure to TCPs or to organochlorine chemicals that are metabolised and excreted as TCPs. ${ }^{5}$ Individual exposure can be reliably estimated by measuring urinary TCPs. ${ }^{6}$

Both experimental studies and epidemiological studies indicate that some organochlorine chemicals such as dichlorodiphenyltrichloroethane (DDT) and dichlorodiphenyldichloroethylene (DDE) are neurodevelopmental toxicants. ${ }^{7} 8$ Several reviews have systemically discussed the developmental neurotoxicity of organochlorine chemicals. ${ }^{9} 10$ Brain development is a complex process involving cell proliferation, migration, differentiation and cell death, and can be disrupted by external environmental agents. A child's central nervous system may be particularly vulnerable to neurotoxicity from environmental agents since it is still developing and environmental agents such as TCPs may easily disturb the process by changing neurotransmitter systems and altering intracellular signalling processes resulting in behavioural impairments. ${ }^{11}$ However, a search of PubMed and Google Scholar databases showed no previously published studies investigating the neurodevelopmental toxicity of TCPs in humans.

Attention deficit hyperactivity disorder (ADHD) is a neurodevelopmental disorder characterised by pervasive inattention and/or hyperactivityimpulsivity causing significant functional impairments. ${ }^{12}{ }^{13}$ The prevalence of $\mathrm{ADHD}$ ranges from $3 \%$ to $8 \%$ in US school-aged children, accounting 
for 4-5 million $\mathrm{ADHD}$ cases among children aged 4-17 years, and has dramatically increased during the past two decades. ${ }^{14-16}$ Despite the increasing prevalence, the aetiology and biological mechanisms of ADHD. Known risk factors associated with increased risk of $\mathrm{ADHD}$ in children include genetic factors and a wide range of environmental toxins including lead, mercury and environmental tobacco smoke (ETS). ${ }^{17-19}$

However, PubMed and Google Scholar searches combining the $\mathrm{MeSH}$ key terms, 'attention-deficit hyperactivity disorder', 'ADHD', 'trichlorophenols' and 'TCP', and review of the references of relevant articles identified no published studies on TCPs and $\mathrm{ADHD}$ in the peer-reviewed literature. Therefore, we used the 1999-2004 National Health and Nutrition Examination Survey (NHANES) data to evaluate the associations between urinary TCPs and ADHD among US children.

\section{METHODS AND MATERIALS Study population}

The NHANES is a cross-sectional household survey that collects extensive health information in face-to-face interviews and from medical examinations among a nationally representative sample of the non-institutionalised US civilian population, obtained with a complex, multi-stage probability sample. ${ }^{20}$ We selected all children between 6 and 15 years of age; we chose this age range because of the availability of data on urinary TCPs and other important study covariates. A total of 2546 children, including 850 children from the 1999-2000 NHANES data, 1035 children from the 2001-2002 NHANES data and 661 children from the 2003-2004 NHANES data, were eligible for this study.

\section{Assessment of ADHD}

We used parental report of their child's diagnosis with ADHD as the primary dependent variable. The ascertainment of children's $\mathrm{ADHD}$ depended on his/her parent or guardian's response to the question, "Has a doctor or health professional ever told [you/SP] that $[\mathrm{you} / \mathrm{s} / \mathrm{he} / \mathrm{SP}]$ had attention deficit disorder?" (where 'SP' referred to 'sample person') which was located in the medical history section of the household questionnaires. If the parent or guardian answered "yes" to the question, the child was categorised as a prevalent case of ADHD.

\section{Measurement of urinary TCPs}

Information on urinary TCPs was obtained from the NHANES data. Per NHANES protocol, urine specimens were collected from subjects in standard urine collection cups. The specimens were labelled, frozen immediately at $-20^{\circ} \mathrm{C}$ and stored on dry ice until analysis. A Sciex API 4000 mass spectrometer was used in negative ion APCI mode. The negative fragment ions were used for quantification of the urinary concentrations of 2,4,5-TCP and 2,4,6-TCP. Details regarding urine specimen collection, storage, handling and testing have been described elsewhere. ${ }^{21} 22$ The creatinine-corrected urinary concentrations of 2,4,5-TCP and 2,4,6-TCP were calculated for further analysis. Creatinine was measured in all urine samples through automated colorimetric determination on a Berman Synchron CX3 clinical analyser (Beckman Instruments, Brea, California, USA) at the University of Minnesota's Fairview Medical Center. $^{23}$ The urinary TCPs $(\mu \mathrm{g} / \mathrm{l})$ were divided by urinary creatinine $(\mathrm{mg} / \mathrm{dl})$ and 0.01 , and expressed as microgram per gram $(\mu \mathrm{g} / \mathrm{g})$ of creatinine.

\section{Covariates}

Covariates were chosen on the basis of their observed associations with $\mathrm{ADHD}$ and/or findings from previous studies. Demographic variables included child's age, gender, race/ethnicity and household socioeconomic status. Specifically, child race/ethnicity included the categories non-Hispanic white, non-Hispanic black, Mexican American and other (including other Hispanic and multiracial) groups. Child's household socioeconomic status was measured by the poverty-to-income ratio (PIR), which is the ratio of family income to the family poverty threshold for the year of the interview and categorised as three groups (ie, $<1,1-2$ and $\geq 2$ ). PIR values below 1.00 are below the official poverty threshold, while PIR values of 1.00 or greater indicate income above the poverty level. ${ }^{24}$ In addition, a review of the literature suggested that low birth weight, lead exposure, and prenatal and postnatal exposure to ETS were potential confounders. ${ }^{18} 192526$ In this analysis, the children's serum cotinine levels, used as a biomarker of postnatal ETS exposure, were obtained from the NHANES data and categorised into tertiles. Children's blood lead levels, measured using inductively coupled plasma mass spectrometry, were also available in the dataset and categorised into tertiles. Lastly, child birth weight, defined as low birth weight (yes/no) and prenatal ETS (ie, prenatal maternal smoking: yes/no) were obtained through personal interview of an adult proxy respondent in the child's family.

\section{Statistical analysis}

Descriptive analyses, such as two-sided Student t tests and Wald $\chi^{2}$ analysis were performed using bivariable analysis of the associations of ADHD with the covariates. Variables associated with ADHD in descriptive analyses $(p<0.2)$ were selected for the multiple variable logistic regression model. The Akaike information criterion (smaller values indicate a better fit) was used to determine the best model in our analysis. In this analysis, data from three data cycles were merged together to represent the US children aged 6-15 years after adjusting for the final weight using the method described below. The cut-off points of urinary TCPs from the entire population rather than those from each data cycle were used to define the groups of urinary TCP levels because similar methods were used to detect TCPs in each cycle. Specifically, children with urinary TCPs below the limit of detection (LOD) were the reference group and those with urinary TCPs above the LOD were categorised into two groups using the urinary concentration median as the cut-off point: less than the median (ie, a low level group) or above the median (ie, a high level group).

All statistical analyses were performed using SAS 9.1 software survey procedures, which account for the complex sampling design used in NHANES. The sample weights, stratification and clustering design variables were incorporated into all SAS survey procedures to ensure the correct estimation of sampling error. A 6-year subsample weight was calculated for the combined 1999-2004 data by following the NHANES analytical and reporting guidelines by assigning two thirds of the subsample weight for 1999-2002 if a participant was sampled in 1999-2002 and one third of the subsample weight for those sampled in 2003-2004. ${ }^{27}$ This calculated weight was used to analyse the combined 6-year data of NHANES 1999-2004.

This study conformed with all applicable requirements of the USA and/or international regulations for research on human subjects. The research protocol was reviewed and approved by the University of Florida Institutional Review Board.

\section{RESULTS}

The distributions of selected characteristics between ADHD cases and controls among children aged 6-15 years are presented in table 1 . Of the 2556 eligible children aged 6-15 years, 2539 
Table 1 Weighted frequency of selected characteristics for children aged 6-15 years with and without parental report of attention deficit hyperactivity disorder (ADHD), NHANES, 1999-2004

\begin{tabular}{|c|c|c|}
\hline \multirow[b]{2}{*}{ Characteristics } & \multicolumn{2}{|c|}{ Parent-reported ADHD } \\
\hline & $\begin{array}{l}\text { Yes }(n=200) \\
\text { N }(95 \% \mathrm{Cl})\end{array}$ & $\begin{array}{l}\text { No }(n=2339) \\
\text { N }(95 \% \text { Cl) }\end{array}$ \\
\hline \multicolumn{3}{|l|}{ Gender* } \\
\hline Female & 25.1 (17.1 to 33.2$)$ & $50.2(47.4$ to 53.0$)$ \\
\hline Male & 74.9 (66.8 to 82.9$)$ & 49.8 (47.0 to 52.6$)$ \\
\hline \multicolumn{3}{|l|}{ Race/ethnicity* } \\
\hline Non-Hispanic white & 71.7 (62.8 to 80.7$)$ & $59.6(54.2$ to 65.1$)$ \\
\hline Non-Hispanic black & 13.0 (9.5 to 16.6$)$ & $15.7(12.4$ to 19.1$)$ \\
\hline Mexican American & 4.7 (2.7 to 6.7$)$ & $12.4(9.3$ to 15.4$)$ \\
\hline Other & 10.5 (2.6 to 18.5$)$ & 12.2 (7.8 to 16.5$)$ \\
\hline \multicolumn{3}{|l|}{ Poverty-to-income ratio } \\
\hline$<1$ & 27.1 (18.5 to 35.7$)$ & 22.2 (19.8 to 24.6$)$ \\
\hline $1-2$ & 28.2 (20.0 to 36.4$)$ & $25.8(22.5$ to 29.1$)$ \\
\hline$\geq 2$ & 44.6 (34.6 to 54.7 ) & $52.0(48.5$ to 55.4$)$ \\
\hline \multicolumn{3}{|l|}{ Blood lead levels $(\mu \mathrm{g} / \mathrm{dl})$} \\
\hline 1st tertile $(\leq 1.0)$ & 39.0 (27.7 to 50.4$)$ & 42.5 (38.1 to 46.9$)$ \\
\hline 2nd tertile $(1.0-1.7)$ & 30.6 (21.6 to 39.5$)$ & 28.6 (25.7 to 31.5$)$ \\
\hline 3rd tertile $(\geq 1.7)$ & 30.4 (19.0 to 41.8$)$ & 28.9 (25.5 to 32.3$)$ \\
\hline \multicolumn{3}{|l|}{ Maternal prenatal tobacco use $\mathrm{e}^{*}$} \\
\hline Yes & 33.5 (23.7 to 43.2$)$ & 16.7 (13.8 to 19.6$)$ \\
\hline No & 66.5 (56.8 to 76.2$)$ & 83.3 (80.4 to 86.2 ) \\
\hline \multicolumn{3}{|l|}{ Infant birth weight } \\
\hline$<2500 \mathrm{~g}$ & $13.2(6.2$ to 20.1$)$ & $6.9(5.9$ to 8.0$)$ \\
\hline$\geq 2500 \mathrm{~g}$ & 86.8 (79.8 to 93.8$)$ & $93.0(92.0$ to 94.1$)$ \\
\hline \multicolumn{3}{|l|}{ Serum cotinine levels $(\mathrm{ng} / \mathrm{ml})^{*}$} \\
\hline 1st tertile $(\leq 0.035)$ & 21.1 (11.4 to 30.9$)$ & 36.8 (31.7 to 41.9$)$ \\
\hline 2nd tertile $(0.035-0.283)$ & 23.0 (15.5 to 30.4$)$ & $30.0(26.6$ to 33.4$)$ \\
\hline 3rd tertile $(\geq 0.283)$ & 55.9 (47.3 to 64.5$)$ & $33.2(29.7$ to 36.70 \\
\hline Child age (years)* & $11.2(10.7$ to 11.6$)$ & $10.4(10.2$ to 10.6$)$ \\
\hline
\end{tabular}

*p value from a Wald $\chi^{2}$ test is less than 0.05

(200 ADHD cases and 2339 controls) were included in the final analysis. Seventeen children were excluded from the analysis because of missing data on the outcome variable. In the bivariate analyses, a higher prevalence of ADHD was found among boys $(\mathrm{p}<0.0001)$, non-Hispanic white children $(\mathrm{p}<0.0001)$, children in older age groups $(p=0.0012)$, children exposed to prenatal maternal smoking $(p=0.005)$ and children with higher serum cotinine levels $(p=0.0005)$. The parents of low birthweight children were more likely to report that their child had ADHD than parents of normal birthweight children $(p=0.09)$.

Table 2 describes the distributions of the creatinine-corrected concentrations of urinary TCPs among children 6-15 years old. The proportion of children with urinary 2,4,6-TCP levels below the LOD increased in the most recent data cycle. However, this was not observed for urinary 2,4,5-TCP. Overall, the range of urinary TCPs among children 6-15 years of age in 2003-2004 was smaller than in 1999-2002.
The associations between urinary $\mathrm{TCP}$ and $\mathrm{ADHD}$ are described in table 3 . The cut-off points for the overall median concentrations of urinary 2,4,6-TCP and 2,4,5-TCP were $3.58 \mu \mathrm{g} /$ $\mathrm{g}$ and $1.03 \mu \mathrm{g} / \mathrm{g}$, respectively. In multivariable analysis, we found that exposure to 2,4,6-TCP was significantly associated with ADHD. Children with low $(<3.58 \mu \mathrm{g} / \mathrm{g})$ or high $(\geq 3.58 \mu \mathrm{g} / \mathrm{g})$ levels of urinary 2,4,6-TCP were more likely to have a parental report of a diagnosis of ADHD compared with children with $2,4,6$ TCP levels below the LOD (OR 1.54, 95\% CI 0.97 to 2.43 and OR $1.77,95 \%$ CI 1.18 to 2.66 , respectively) after adjusting for demographic factors, early childhood factors and other environmental toxins. A dose-response relationship between urinary 2,4,6-TCP and ADHD was also observed ( $p$ for trend $=0.006$ ). However, we did not find a significant association between exposure to 2,4,5-TCP and ADHD. Additionally, we defined the exposure groups of urinary TCPs using the cut-off points from each data cycle. We repeated the analysis and the conclusion remained similar (ie, no significant effects for 2,4,5-TCP but a significant effect for 2,4,6-TCP). We also evaluated the associations by including additional covariates, such as dimethylthiophosphate exposure, in the models and found similar results (data are not presented).

Furthermore, we examined the interactions between urinary TCPs and other covariates including age, gender, race/ethnicity, low birth weight and prenatal passive smoking. No significant interactions were found (data are not presented).

\section{DISCUSSION}

Our intention was to evaluate the neurological health effects of low levels of 2,4,5-TCP and 2,4,6-TCP among school-aged children. We found that exposure to 2,4,6-TCP was associated with parental report of a diagnosis of $\mathrm{ADHD}$. We also demonstrated a significant dose-response relationship between exposure to 2,4,6-TCP and ADHD. In contrast, we did not find a significant association between exposure to 2,4,5-TCP and ADHD. These findings suggest that 2,4,6-TCP may have different health effects than 2,4,5-TCP, which is consistent with patterns of association previously reported in the literature. Experimental evidence suggests that 2,4,6-TCP but not 2,4,5-TCP is associated with increased risks of multiple cancers. ${ }^{28} 29$

Both 2,4,6-TCP and 2,4,5-TCP are members of a family of organochlorine pesticides which are a large class of multipurpose chlorinated hydrocarbon chemicals that break down slowly in the environment and accumulate in fatty tissue. The presence of urinary TCPs indicates direct exposure to these chemicals or other organochlorine pesticides including hexachlorobenzene ( $\mathrm{HCB})$ and hexachlorocyclohexanes $(\mathrm{HCH})$ which metabolise to TCPs in humans. ${ }^{30} 31$ Although many organochlorine pesticides are no longer widely used in the USA, children can be exposed to these chemicals through diet and contaminated air because of the intensive use of these chemicals in the past, and their lipophilic and chemically stable characters. ${ }^{32}$ Exposure to

Table 2 Distribution of creatinine-corrected concentrations $(\mu \mathrm{g} / \mathrm{g})$ of urinary TCPs among children aged 6-15 years in NHANES, 1999-2004

\begin{tabular}{|c|c|c|c|c|c|c|c|c|}
\hline \multirow[b]{3}{*}{ Data cycle } & \multicolumn{4}{|c|}{ 2,4,5-TCP } & \multicolumn{4}{|c|}{ 2,4,6-TCP } \\
\hline & \multirow[b]{2}{*}{$\mathbf{N}$} & \multirow[b]{2}{*}{$<\operatorname{LOD}(\%)$} & \multicolumn{2}{|l|}{$\geq L O D$} & \multirow[b]{2}{*}{$\mathbf{N}$} & \multirow[b]{2}{*}{ <LOD (\%) } & \multicolumn{2}{|l|}{$\geq \mathbf{L O D}$} \\
\hline & & & Median $(\mu \mathrm{g} / \mathrm{g})$ & Range $(\mu \mathrm{g} / \mathrm{g})$ & & & Median $(\mu \mathrm{g} / \mathrm{g})$ & Range $(\mu \mathrm{g} / \mathrm{g})$ \\
\hline $2001-2002$ & 1013 & 95.2 & 6.17 & $0.51-124.7$ & 1018 & 33.3 & 4.76 & $0.68-106.6$ \\
\hline $2003-2004$ & 657 & 61.3 & 0.12 & $0.03-5.2$ & 657 & 62.1 & 0.65 & $0.16-22.2$ \\
\hline Overall & 2518 & 70.8 & 1.03 & $0.25-124.7$ & 2520 & 33.6 & 3.58 & $0.16-1772.2$ \\
\hline
\end{tabular}

LOD, below the limit of detection; TCP, trichlorophenol. 
Table 3 ORs and 95\% Cls from logistic regression analysis for the association between urinary trichlorophenols and attention deficit hyperactivity disorder among children aged $6-15$ years, NHANES, 1999-2004

\begin{tabular}{|c|c|c|c|c|}
\hline Chemicals & Total sample (n) & Cases (n) & Crude odd ratios & Adjusted odd ratios* \\
\hline \multicolumn{5}{|l|}{ 2,4,5-ТCP } \\
\hline Below the LOD & 1776 & 145 & Referent group & Referent group \\
\hline$<$ Median $(1.03 \mu \mathrm{g} / \mathrm{g})$ & 367 & 30 & $1.27(0.78$ to 2.06$)$ & 1.01 (0.47 to 2.20$)$ \\
\hline$\geq$ Median $(1.03 \mu \mathrm{g} / \mathrm{g})$ & 368 & 24 & 0.89 (0.48 to 1.65$)$ & 0.98 (0.48 to 1.99$)$ \\
\hline $\mathrm{p}$ for trend & - & - & 0.72 & 0.95 \\
\hline \multicolumn{5}{|l|}{ 2,4,6-TCP } \\
\hline Below the LOD & 846 & 56 & Referent group & Referent group \\
\hline$<$ Median $(3.58 \mu \mathrm{g} / \mathrm{g})$ & 834 & 84 & 1.84 (1.25 to 2.72$)$ & 1.54 (0.97 to 2.43$)$ \\
\hline$\geq$ Median $(3.58 \mu \mathrm{g} / \mathrm{g})$ & 834 & 59 & 1.56 (1.12 to 2.16$)$ & 1.77 (1.18 to 2.66$)$ \\
\hline $\mathrm{p}$ for trend & - & - & 0.008 & 0.006 \\
\hline
\end{tabular}

these chemicals may occur early in life. ${ }^{33}$ Although information on the potential health effects of $\mathrm{HCB}$ and $\mathrm{HCH}$ on child neurodevelopment is limited, evidence of neurotoxicity in humans exposed to organochlorine compounds including HCB and $\mathrm{HCH}$ has previously been presented. ${ }^{34} 35$ Several studies suggest that prenatal exposure to organochlorine compounds is associated with an increase in $\mathrm{ADHD}$ symptoms among children at 4 years of age. ${ }^{36-39}$ Therefore, the observed association between urinary 2,4,6-TCP and ADHD in our study does not exclude the possibility that the ADHD is caused by HCB and/or $\mathrm{HCH}$ exposures.

The observed association also suggests that direct exposure to 2,4,6-TCP directly affects child neurodevelopment. However, there is limited information on the neurological health effects of 2,4,6-TCP in humans. Because 2,4,6-TCP is one of the major chlorinated organic compounds which are formed during water disinfection, children can be exposed to the chemical through drinking water. ${ }^{40}$ Confirmation of a direct health effect of 2,4,6-TCP on child behavioural development would underscore the importance of further examining the potential neurological health effects of prenatal and postnatal exposure to 2,4,6-TCP through chlorinated drinking water.

Our study has several strengths. First, a large population-based sample was used to examine the neurological effects of TCPs and valuable information on the effect of background exposure to TCPs on child neurodevelopment in the general population was collected. Second, we used biomarkers in urine to measure exposures to organochlorine compounds. This method provided an overall measurement of chemical exposures from all sources and a more accurate measurement of body burden of exposure. Third, a wide of range of potential confounders including demographic factors and other environmental toxins such as lead and ETS, was included in the models. Moreover, the exposures to environmental toxins were measured using biomarkers in serum, which could minimise misclassification errors. Fourth, to test the reliability of our estimation we constructed several models with different covariates and found consistent results. Our analyses provided reasonably consistent findings, which are strengthened by an observed dose-response relationship.

Several potential limitations should be considered when interpreting the results of our study. First, although the use of urine as a matrix to determine biomarkers of exposure is especially suitable for compounds such as TCPs and other metabolites, urinary levels may reflect recent exposure. In addition, the large variation in TCP levels between data cycles suggests data errors during testing and/or data processing unless urinary TCPs varied greatly between different cycles. Second, since this is a crosssectional study, we cannot interpret our results as providing evidence of a causal relationship between early exposure to TCPs and subsequent risk of developing $\mathrm{ADHD}$. Third, ascertainment of $\mathrm{ADHD}$ was solely based on parental report without confirmation of diagnosis through medical record abstraction or administration of the Diagnostic and Statistical Manual of Mental Disorders, Fourth Edition (DSM-IV). Administration of the DSM would provide information on ADHD subtypes and behavioural symptom counts, and increase specificity in detecting behavioural problems. Although data from the DSM-based $\mathrm{ADHD}$ diagnostic instruments are available in NHANES, there is limited access to this information. In addition, misclassification of $\mathrm{ADHD}$ from parental report would mostly likely result in underestimation of the true association between TCPs and ADHD. Future prospective cohort studies with better measures of TCP exposure and better ascertainment of $\mathrm{ADHD}$ are needed to validate our findings. Fourth, the data available on covariates such as socioeconomic status and early childhood factors were obtained through personal interview of a proxy respondent of a child's family and may be inaccurate. Finally, although many important covariates and potential confounders were included in our analysis, we were unable to adjust for the potential effects of other important covariates including parental education, breastfeeding, genetic factors, psychosocial stressors, other prenatal and/or postnatal environmental factors such as prenatal alcohol drinking, exposure to polychlorinated biphenyls, dioxins, phthalate and bisphenol A, and parental psychopathology.

Nevertheless, our results suggest that exposure to organochlorine compounds may be associated with increased risk of $\mathrm{ADHD}$ among school-aged children. Our findings underscore the potential behavioural health effects of these chemicals and highlight the need to strengthen public health efforts to reduce prenatal and postnatal exposure to these compounds, especially in countries where organochlorine pesticides are still commonly used.

\section{Competing interests None.}

Ethics approval This study was conducted with the approval of the University of Florida IRB.

Provenance and peer review Not commissioned; externally peer reviewed.

\section{REFERENCES}

1. Tai C, Jiang G. Dechlorination and destruction of 2,4,6-trichlorophenol and pentachlorophenol using hydrogen peroxide as the oxidant catalyzed by molybdate ions under basic condition. Chemosphere 2005;59:321-6.

2. ATSDR. Toxicological profile for chlorophenols [online]. 1999. http://www.atsdr.cdc. gov/toxprofiles/tp107.html (accessed 21 May 2010). 
3. Macholz RM, Knoll R, Lewerenz HJ, et al. Metabolism of alpha-hexachlorocyclohexane. Free metabolites in urine and organs of rats. Xenobiotica 1982;12:227-31.

4. Lechner ML, Somogyi MA, Biro ML. Detection and determination of chlorophenols and chlorophenoxyacetic acids by instrumental analysis. Talanta 1966:13:581-7.

5. Nichkova M, Marco MP. Biomonitoring human exposure to organohalogenated substances by measuring urinary chlorophenols using a high-throughput screening (HTS) immunochemical method. Environ Sci Technol 2006:40:2469-77.

6. Angerer J, Maass R, Heinrich R. Occupational exposure to hexachlorocyclohexane. VI. Metabolism of gamma-hexachlorocyclohexane in man. Int Arch Occup Environ Health 1983;52:59-67.

7. Eskenazi B, Marks AR, Bradman A, et al. In utero exposure to dichlorodiphenyltrichloroethane (DDT) and dichlorodiphenyldichloroethylene (DDE) and neurodevelopment among young Mexican American children. Pediatrics 2006:118:233-41.

8. Eriksson $\mathbf{P}$, Nordberg A. The effects of DDT, DDOH-palmitic acid, and a chlorinated paraffin on muscarinic receptors and the sodium-dependent choline uptake in the central nervous system of immature mice. Toxicol Appl Pharmacol 1986;85:121-7

9. Eskenazi B, Rosas LG, Marks AR, et al. Pesticide toxicity and the developing brain. Basic Clin Pharmacol Toxicol 2008:102:228-36.

10. Banerjee TD, Middleton F, Faraone SV. Environmental risk factors for attentiondeficit hyperactivity disorder. Acta Paediatr 2007;96:1269-74.

11. Kodavanti PR. Neurotoxicity of persistent organic pollutants: possible mode(s) of action and further considerations. Dose Response 2006;3:273-305.

12. Centers for Disease Control and Prevention(CDC). Mental health in the United States. Prevalence of diagnosis and medication treatment for attention-deficit/ hyperactivity disorder-United States, 2003. MMWR Morb Mortal Wkly Rep 2005:54:842-7.

13. Xiang H, Stallones L, Chen G, et al. Nonfatal injuries among US children with disabling conditions. Am J Public Health 2005;95:1970-5.

14. Mandell DS, Thompson WW, Weintraub ES, et al. Trends in diagnosis rates for autism and ADHD at hospital discharge in the context of other psychiatric diagnoses. Psychiatr Serv 2005:56:56-62.

15. Rowland AS, Lesesne CA, Abramowitz AJ. The epidemiology of attention-deficit/ hyperactivity disorder (ADHD): a public health view. Ment Retard Dev Disabil Res Rev 2002:8:162-70.

16. Bloom B, Cohen RA. Summary health statistics for U.S. children: National Health Interview Survey, 2006. Vital Health Stat 2007;10:1-79.

17. Watanabe C, Satoh H. Evolution of our understanding of methylmercury as a health threat. Environ Health Perspect 1996;104(Suppl 2):367-79.

18. Froehlich TE, Lanphear BP, Auinger $P$, et al. Association of tobacco and lead exposures with attention-deficit/hyperactivity disorder. Pediatrics 2009;124 e1054-63.

19. Braun JM, Kahn RS, Froehlich T, et al. Exposures to environmental toxicants and attention deficit hyperactivity disorder in U.S. children. Environ Health Perspect 2006;114:1904-9

20. CDC. National Health and Nutrition Examination Survey. Hyattsville, MD: National Center for Health Statistics (NCHS), 2006. http://www.cdc.gov/nchs/nhanes/ about nhanes.htm (accessed 5 May 2010).

21. CDC. Laboratory Procedure Manual: Environmental Pesticides, 2005. http://www. cdc.gov/nchs/data/nhanes/nhanes_03_04/I24eph_c_met_phenols.pdf laccessed 6 May 2010).
22. Ye X, Kuklenyik Z, Needham LL, et al. Automated on-line column-switching HPLC-MS/MS method with peak focusing for the determination of nine environmenta phenols in urine. Anal Chem 2005;77:5407-13.

23. CDC. Laboratory Procedure Manual: Cotinine, 2001. http://www.cdc.gov/nchs/data/ nhanes/nhanes_01_02/106_b_met_cotinine.pdf. (accessed 11 Feb 2011).

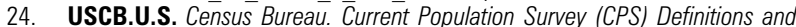
Explanations, 2003. http://www.census.gov/population/www/cps/cpsdef.html (accessed 20 May 2010).

25. Weitzman $\mathbf{M}$, Gortmaker S, Sobol A. Maternal smoking and behavior problems of children. Pediatrics 1992;90:342-9.

26. Gospe SM Jr, Zhou SS, Pinkerton KE. Effects of environmental tobacco smoke exposure in utero and/or postnatally on brain development. Pediatr Res 1996;39:494-8.

27. CDC. NHANES. Analytic Guidelines, June 2004 Version. Hyattsville, MD: National Center for Health Statistics (NCHS), 2004. http://www.cdc.gov/nchs/data/nhanes/ nhanes_general_guidelines_june_04.pdf (assessed 5 May 2010).

28. Rasanen L, Hattula ML, Arstila AU. The mutagenicity of MCPA and its soil metabolites, chlorinated phenols, catechols and some widely used slimicides in Finland. Bull Environ Contam Toxicol 1977;18:565-71.

29. Kinae N, Hashizume T, Makita T, et al. Studies on the toxicity of pulp and paper-mill effluents .1. mutagenicity of the sediment samples derived from kraft paper-mills. Water Res 1981;15:17-24.

30. Renner G, Schuster KP. 24,5-Trichlorophenol, a new urinary metabolite of hexachlorobenzene. Toxicol Appl Pharmacol 1977;39:355-6.

31. Freal JJ, Chadwick RW. Metabolism of hexachlorocyclohexane to chlorophenols and effect of isomer pretreatment on lindane metabolism in rat. J Agric Food Chem 1973;21:424-7.

32. Toppari J, Larsen JC, Christiansen $\mathrm{P}$, et al. Male reproductive health and environmental xenoestrogens. Environ Health Perspect 1996;104(Suppl 4):741-803

33. Castorina R, Bradman A, Fenster L, et al. Comparison of current-use pesticide and other toxicant urinary metabolite levels among pregnant women in the CHAMACOS cohort and NHANES. Environ Health Perspect 2010;118:856-63.

34. Muller D, Klepel H, Macholz RM, et al. Electroneurophysiological studies on neurotoxic effects of hexachlorocyclohexane isomers and gammapentachlorocyclohexene. Bull Environ Contam Toxicol 1981;27:704-6.

35. Peters HA, Gocmen A, Cripps DJ, et al. Epidemiology of hexachlorobenzene-induced porphyria in Turkey: clinical and laboratory follow-up after 25 years. Arch Neurol 1982;39:744-9.

36. Ribas-Fito N, Torrent M, Carrizo D, et al. Exposure to hexachlorobenzene during pregnancy and children's social behavior at 4 years of age. Environ Health Perspect 2007; 115:447-50.

37. Darvill T, Lonky E, Reihman J, et al. Prenatal exposure to PCBs and infant performance on the fagan test of infant intelligence. Neurotoxicology 2000:21:1029-38.

38. Sagiv SK, Thurston SW, Bellinger DC, et al. Prenatal organochlorine exposure and behaviors associated with attention deficit hyperactivity disorder in school-aged children. Am J Epidemiol 2010;171:593-601.

39. Jacobson JL, Jacobson SW. Prenatal exposure to polychlorinated biphenyls and attention at school age. J Pediatr 2003;143:780-8.

40. Michalowicz J. The occurrence of chlorophenols, chlorocatechols and chlorinated methoxyphenols in drinking water of the largest cities in Poland. Pol J Environ Stud 2005; 14:327-33 